\title{
Approximate controllability of impulsive neutral functional differential equations with state-dependent delay via fractional operators
}

\author{
N. Y. Nadaf \\ Department of Mathematics, \\ Karunya University, Karunya Nagar, \\ Coimbatore-614 114, Tamil Nadu, India. \\ nadaf_nabisab@yahoo.com
}

\author{
M. Mallika Arjunan \\ Department of Mathematics, \\ C. B. M. College, Kovaipudur, \\ Coimbatore- 641 042, Tamil Nadu, India. \\ arjunphd07@yahoo.co.in
}

\begin{abstract}
In this article, the problem of approximate controllability for nonlinear impulsive neutral differential systems with state-dependent delay is studied under the assumption that the corresponding linear control system is approximately controllable. Using Schauder's fixed point theorem and fractional powers of operators with semigroup theory, sufficient conditions are formulated and proved.
\end{abstract}

\section{Keywords:}

Approximate controllability, Impulsive neutral functional differential equations, Semigroup theory, State-dependent delay, Fixed point..

\section{INTRODUCTION}

In this paper, we study the approximate controllability of the impulsive neutral functional differential equation with state-dependent delay in the form:

$$
\begin{aligned}
\frac{d}{d t}\left[x(t)+g\left(t, x_{t}\right)\right] & =A x(t)+B u(t)+f\left(t, x_{\rho\left(t, x_{t}\right)}\right), \\
t & \in J=[0, b] \\
x_{0} & =\phi \in \mathcal{B}, \\
\Delta x\left(t_{k}\right) & =I_{k}\left(x_{t_{k}}\right), \quad k=1,2, \ldots, m,
\end{aligned}
$$

where $A$ is the infinitesimal generator of a strongly continuous semigroup of bounded linear operators on a Banach space $X, B$ is a bounded linear operator from a Banach space $U$ into $X$. The notation $x_{s}$ represents the function defined by $x_{s}:(-\infty, 0] \rightarrow$ $X, \quad x_{s}(\theta)=x(s+\theta)$, belongs to some abstract phase space $\mathcal{B}$ described axiomatically and $\rho: J \times \mathcal{B} \rightarrow(-\infty, b]$ is a continuous function. Further $f, g: J \times \mathcal{B} \rightarrow X$, and $I_{k}(\cdot): \mathcal{B} \rightarrow X$ are appropriate functions, the control $u(\cdot) \in L_{2}(J, U)$, a Banach space of admissible control functions. Here $0<t_{1}<t_{2}<\cdots<t_{m}<b$ are pre-fixed numbers and $\Delta \xi(t)$ represents the jump $\xi$ at time $t$ which is defined by $\Delta \xi(t)=\xi\left(t^{+}\right)-\xi\left(t^{-}\right)$.

The theory of impulsive differential equations has become an important area of investigation in recent years. Relative to this the- ory, we only refer the interested reader to [1] and the monographs [2, 3, 4].

The concept of controllability is an important property of a control system which plays an important role in many control problems such as stabilization of unstable systems by feedback control. Therefore, in recent years controllability problems for various types of linear and nonlinear deterministic and stochastic dynamic systems have been studied by many authors, see for instance [5, 6, 7, 8, 9, 10, 11]. In particular, approximate controllable systems are more prevalent and very often approximate controllability is completely adequate in applications. There are many papers on the approximate controllability of the various types of nonlinear systems under different conditions, see for instance [12, 13, 14, 15, 17, 18] and references therein. Approximate controllability for semilinear deterministic and stochastic control systems can be found in Mahmudov [19]. More recently, Sakthivel et al. [14] derived a set of sufficient conditions for the approximate controllability of nonlinear deterministic and stochastic systems with unbounded delay by using the Schauder's fixed point theorem.

On the other hand, delay differential equations arise in many physical and biological applications, but often demand the use of nonconstant or state-dependent delays. These equations are frequently called equations with state-dependent delay. In recent years, the analysis of differential equations with state-dependent delay have received much attention, see for instance [20]. Very recently, existence of mild solutions to impulsive abstract neutral functional differential equations with state dependent delay has been established; see for instance [21] and the references therein. However, it should be emphasized that the approximate controllability of neutral differential equations with state-dependent delay is not yet sufficiently elaborated, compared to that of classical differential equations. In this regard in fact, it is necessary and important to study approximate controllability problems for nonlinear neutral systems with state-dependent delay. To the best of the authors knowledge, the approximate controllability of nonlinear neutral differential equations with state-dependent delay of the form $(1)-\sqrt{3}$ has not been studied yet in the existing literature. Motivated by this consideration, in this paper we establish sufficient conditions for the approximate controllability of the nonlinear impulsive neutral differential 
systems with state-dependent delay by using the Schauder's fixxed point theorem and fractional powers of operators with semigroup theory.

The remainder of this paper is organized as follows. In section 2 , we give some notations, definitions and basic results about semigroup theory and approximate controllability. In section 3, first we give the existence of solutions for the problem (1) - (3) by using the Schauder's fixed point theorem, and then sufficient conditions for approximate controllability of impulsive neutral differential systems with state-dependent delay are established.

\section{PRELIMINARIES}

In this section, we recall some notations, definitions and lemmas which are used in this paper.

A function $x:[\sigma, \mu] \rightarrow X$ is said to be a normalized piecewise continuous function on $[\sigma, \mu]$ if $x$ is piecewise continuous and left continuous on $[\sigma, \mu]$. We denote by $P C([\sigma, \mu], X)$ the space of normalized piecewise continuous functions from $[\sigma, \mu]$ into $X$. In particular, we introduce the space $P C$ formed by all normalized piecewise continuous functions $x:[0, b] \rightarrow X$ such that $x(\cdot)$ is continuous at $t \neq t_{k}, x\left(t_{k}^{-}\right)=x\left(t_{k}\right)$ and $x\left(t_{k}^{+}\right)$exists, for $k=$ $1, \ldots, m$. It is clear that $P C$ endowed with the norm $\|x\|_{P C}=$ $\sup _{s \in J}\|x(s)\|$ is a Banach space.

Let $A: D(A) \rightarrow X$ be the infinitesimal generator of an analytic semigroup $T(t), t \geq 0$, of bounded linear operators on a Banach space $X$. Let $0 \in \rho(A)$, then it is possible to define the fractional power $(-A)^{\alpha}$, for $0<\alpha \leq 1$, as closed linear operator on its domain $D(-A)^{\alpha}$. Furthermore, the subspace $D(-A)^{\alpha}$ is dense in $X$, and the expression

$$
\|x\|_{\alpha}=\left\|(-A)^{\alpha} x\right\|, \quad x \in D(-A)^{\alpha}
$$

defines a norm on $D(-A)^{\alpha}$.

Furthermore, we have the following properties appeared in [23].

\section{LEMMA 1. ([23]). The following properties hold.}

(i) If $0<\beta<\alpha \leq 1$, then $X_{\alpha} \subset X_{\beta}$ and the embedding is compact whenever the resolvent operator of $A$ is compact.

(ii) For every $0<\alpha \leq 1$ there exists $C_{\alpha}>0$ such that

$$
\left\|(-A)^{\alpha} R(t)\right\| \leq \frac{C_{\alpha}}{t^{\alpha}}, \quad 0<t \leq b .
$$

In this work we will employ an axiomatic definition of the phase space $\mathcal{B}$, which is similar to those introduced in [23]. Specifically, $\mathcal{B}$ will be a linear space of functions mapping $(-\infty, 0]$ into $X$ endowed with a seminorm $\|\cdot\|_{\mathcal{B}}$ and we will assume that $\mathcal{B}$ satisfies the following axioms:

$\left(A_{1}\right)$ If $x:(-\infty, \sigma+b] \rightarrow X, b>0$, is such that $x_{\sigma} \in \mathcal{B}$ and $\left.x\right|_{[\sigma, \sigma+b]} \in P C([\sigma, \sigma+b], X)$, then for every $t \in[\sigma, \sigma+b)$ the following conditions hold:

(i) $x_{t}$ is in $\mathcal{B}$,

(ii) $\|x(t)\| \leq \tilde{H}\left\|x_{t}\right\|_{\mathcal{B}}$,

(iii) $\left\|x_{t}\right\|_{\mathcal{B}} \leq \tilde{K}(t-\sigma) \sup \{\|x(s)\|: \sigma \leq s \leq t\}+$ $\tilde{M}(t-\sigma)\left\|x_{\sigma}\right\|_{\mathcal{B}}$, where $\tilde{H}>0$ is a constant; $\tilde{K}, \tilde{M}$ : $[0, \infty) \rightarrow[1, \infty), \tilde{K}$ is continuous, $\tilde{M}$ is locally bounded, and $\tilde{H}, \tilde{K}, \tilde{M}$ are independent of $x(\cdot)$.

$\left(A_{2}\right)$ The space $\mathcal{B}$ is complete.

For more details about phase space axioms and examples, we refer the reader to [24].
Let $x_{b}(\phi ; u)$ be the state value of $13-(3)$ at terminal time $b$ corresponding to the control $u$ and the initial value $\phi$. Introduce the set

$$
\Re(b, \phi)=\left\{x_{b}(\phi ; u)(0): u() \in L_{2}(J, U)\right\},
$$

which is called the reachable set of system (1)-(3) at terminal time $b$, its closure in $X$ is denoted by $\overline{(\Re(b, \phi))}$.

Definition 1. A function $x:(-\infty, b] \rightarrow X$ is called $a$ mild solution of the abstract Cauchy problem (1)-(3) if $x_{0}=$ $\phi ; x_{\rho\left(s, x_{s}\right)} \in \mathcal{B}$ for every $s \in J ;$ the function $t \rightarrow A T(t-$ $s) g\left(s, x_{s}\right)$ is integrable on $[0, t)$, for every $t \in[0, b]$, and the integral equation

$$
\begin{aligned}
x(t)= & T(t)[\phi(0)+g(0, \phi)]-g\left(t, x_{t}\right)-\int_{0}^{t} A T(t-s) g\left(s, x_{s}\right) d s \\
& +\int_{0}^{t} T(t-s)\left[B u(s)+f\left(s, x_{\rho\left(s, x_{s}\right)}\right)\right] d s \\
& +\sum_{0<t_{k}<t} T\left(t-t_{k}\right) I_{k}\left(x_{t_{k}}\right), \quad t \in J,
\end{aligned}
$$

is satisfied.

DEFINITION 2. A system (1)-(3) is said to be approximate controllable on the interval $[0, b]$ if $(\Re(b, \phi))$ is dense in $X$, i.e., $\overline{(\Re(b, \phi))}=X$.

It is convenient at this point to define operators

$$
\begin{aligned}
\Gamma_{0}^{b} & =\int_{0}^{b} T(b-s) B B^{*} T^{*}(b-s) d s, \\
R\left(\alpha, \Gamma_{0}^{b}\right) & =\left(\alpha I+\Gamma_{0}^{b}\right)^{-1},
\end{aligned}
$$

where $B^{*}$ denotes the adjoint of $B$ and $T^{*}$ is the adjoint of $T$. It is straight forward that the operator $\Gamma_{0}^{b}$ is a linear bounded operator. Assume the following:

$\left(\mathbf{S}_{1}\right) \alpha R\left(\alpha, \Gamma_{0}^{b}\right) \rightarrow 0$ as $\alpha \rightarrow 0^{+}$in the strong operator topology. It is known from [25] that the assumption $\left(S_{1}\right)$ holds if and only if the linear system

$$
\begin{aligned}
x^{\prime}(t) & =A x(t)+B u(t), \quad t \in J=[0, b], \\
x_{0} & =\phi \in \mathcal{B},
\end{aligned}
$$

is approximate controllable on $J$.

Lemma 2. ([26],Lemma 2.1]). Let $x:(-\infty, b] \rightarrow X$ be a function such that $x_{0}=\phi$ and $\left.x\right|_{J} \in P C$. Then

$\left\|x_{s}\right\|_{\mathcal{B}} \leq\left(M_{a}+J_{0}^{\phi}\right)\|\phi\|_{\mathcal{B}}+K_{a} \sup \{\|x(\theta)\|: \theta \in[0, \max \{0, s\}]\}$, $s \in \mathcal{R}\left(\rho^{-}\right) \cup J$.

where $M_{a}=\sup _{s \in J} M(s), \quad K_{a}=\sup _{s \in J} K(s), \quad J_{0}^{\phi}=$ $\sup _{s \in \mathcal{R}\left(\rho^{-}\right)} J^{\phi}(s)$.

\section{APPROXIMATE CONTROLLABILITY}

To establish our results, we list the following assumptions on system (1)-(3).

$\left(H_{1}\right) \rho: J \times \mathcal{B} \rightarrow(-\infty, b]$ is continuous.

$\left(H_{2}\right)$ Let $\mathcal{R}\left(\rho^{-}\right)=\{\rho(s, \psi):(s, \psi) \in J \times \mathcal{B}, \rho(s, \psi) \leq 0\}$. The function $t \rightarrow \phi_{t}$ is well defined from $\mathcal{R}\left(\rho^{-}\right)$into $\mathcal{B}$ and there exists a continuous and bounded function $J^{\phi}:\left(\rho^{-}\right) \rightarrow(0, \infty)$ such that $\left\|\phi_{t}\right\|_{\mathcal{B}} \leq J^{\phi}(t)\|\phi\|_{\mathcal{B}}$ for every $t \in \mathcal{R}\left(\rho^{-}\right)$. 
$\left(H_{3}\right)$ The function $f: J \times \mathcal{B} \rightarrow X$ satisfies the following conditions:

(i) Let $x:(-\infty, b] \rightarrow X$ be such that $x_{0}=\phi$ and $\left.x\right|_{J} \in$ $P C$. The function $t \rightarrow f\left(t, x_{\rho\left(t, x_{t}\right)}\right)$ is measurable on $J$ and the function $t \rightarrow f\left(s, x_{t}\right)$ is continuous on $\mathcal{R}\left(\rho^{-}\right) \cup J$ for every $s \in J$.

(ii) For each $t \in J$, the function $f(t):, \mathcal{B} \rightarrow X$ is continuous.

(iii) For each $q>0$ there exists a function $\lambda_{q} \in L^{1}\left(J, R^{+}\right)$ such that $\sup _{\|\xi\| \leq q}\|f(t, \xi)\| \leq \lambda_{q}(t)$ for a.e. $t \in J$, and

$$
\liminf _{q \infty 0} \int_{0}^{b} \frac{\lambda_{q}(t)}{q} d t=\Lambda<\infty
$$

$\left(H_{4}\right)$ There exist constants $0<\beta<1, L_{1}, L_{2}, L_{g}$ such that $g$ is $X_{\beta}$-valued, $(-A)^{\beta} g$ is continuous, and

(i) $\left\|(-A)^{\beta} g(t, x)\right\| \leq L_{1}\|x\|_{\mathcal{B}}+L_{2}, \quad t \in J, \quad x \in \mathcal{B}$,

(ii) $\left\|(-A)^{\beta} g\left(t, x_{1}\right)-(-A)^{\beta} g\left(s, x_{2}\right)\right\| \leq L_{g}\left[|t-s|+|| x_{1}-\right.$ $\left.x_{2} \|_{\mathcal{B}}\right], \quad t, s \in J, x_{i} \in \mathcal{B}, i=1,2$ with $\left\|(-A)^{-\beta}\right\|=$ $M_{0},\left\|(-A)^{1-\beta}\right\|=N_{0}$.

$\left(H_{5}\right)$ The maps $I_{k}$ are continuous and there exists a positive constant $d_{k}$ such that $\left\|I_{k}(\xi)\right\| \leq d_{k}$.

$\left(H_{6}\right)$ The semigroup $T(t), t>0$ is compact.

$\left(H_{7}\right)$ The function $f: J \times \mathcal{B} \rightarrow X$ is continuous and uniformly bounded and there exists an $N>0$ such that $\|f(t, \xi)\| \leq N$ for all $(t, \xi) \in J \times \mathcal{B}$.

Now for convenience, we list the following notations

$$
\begin{aligned}
M= & \max \{\|T(t)\|: 0 \leq t \leq b\}, \quad M_{1}=\|B\|, \\
K= & \left\|x_{b}\right\|+M \bar{H}\|\phi\|_{\mathcal{B}}+M M_{0}\left(L_{1}\|\phi\|_{\mathcal{B}}+L_{2}\right)+M \sum_{k=1}^{m} d_{k}, \\
K^{*}= & M \bar{H}\|\phi\|_{\mathcal{B}}+M M_{0}\left(L_{1}\|\phi\|_{\mathcal{B}}+L_{2}\right)+M^{2} M_{1}^{2} \frac{b}{\alpha} K \\
& +M \sum_{k=1}^{m} d_{k} .
\end{aligned}
$$

It will be shown that the system (1)-(3) is approximately controllable, if for all $\alpha>0$ there exists a continuous function $x(\cdot) \in Z$ such that

$$
\begin{aligned}
u(t)= & B^{*} T^{*}(b-t) R\left(\alpha, \Gamma_{0}^{b}\right) p(x(\cdot)), \\
x(t)= & T(t)[\phi(0)+g(0, \phi)]-g\left(t, x_{t}\right) \\
& -\int_{0}^{t} A T(t-s) g\left(s, x_{s}\right) d s+\int_{0}^{t} T(t-s)[B u(s) \\
& \left.+f\left(s, x_{\rho\left(s, x_{s}\right)}\right)\right] d s+\sum_{0<t_{k}<t} T\left(t-t_{k}\right) I_{k}\left(x_{t_{k}}\right), \quad t \in J,
\end{aligned}
$$

where

$$
\begin{aligned}
p(x(\cdot))= & x_{b}-T(b)[\phi(0)+g(0, \phi)]-g\left(b, x_{b}\right) \\
& -\int_{0}^{b} A T(b-s) g\left(s, x_{s}\right) d s \\
& -\int_{0}^{b} T(b-s) f\left(s, x_{\rho\left(s, x_{s}\right)}\right) d s-\sum_{k=1}^{m} T\left(b-t_{k}\right) I_{k}\left(x_{t_{k}}\right) .
\end{aligned}
$$

THEOREM 1. Assume that conditions $\left(H_{1}\right)-\left(H_{7}\right)$ are satisfied. Further, suppose that for all $\alpha>0$

$$
\left(1+M^{2} M_{1}^{2} \frac{b}{\alpha}\right)\left[\left(M_{0}+\frac{C_{1-\beta} b^{\beta}}{\beta}\right) L_{1}+\Lambda M\right] K_{a}<1,
$$

then the system (17)-(3) has a solution on J.

Proof. Let $Z=\{x \in P C: x(0)=\phi(0)\}$ be the space endowed with the uniform convergence topology. On the space $Z$, consider the set $Q=\{x \in Z ;\|x\| \leq r\}$, where $r$ is a positive constant. For $\alpha>0$, define the operator $\psi: Z \rightarrow Z$ by $\psi x(t)=$ $z(t)$, where

$$
\begin{aligned}
v(t)= & B^{*} T^{*}(b-t) R\left(\alpha, \Gamma_{0}^{b}\right) p(x(\cdot)), \\
z(t)= & T(t)[\phi(0)+g(0, \phi)]-g\left(t, \bar{x}_{t}\right) \\
& -\int_{0}^{t} A T(t-s) g\left(s, \bar{x}_{s}\right) d s \\
& +\int_{0}^{t} T(t-s)\left[B v(s)+f\left(s, \bar{x}_{\rho\left(s, \bar{x}_{s}\right)}\right)\right] d s \\
& +\sum_{0<t_{k}<t} T\left(t-t_{k}\right) I_{k}\left(\bar{x}_{t_{k}}\right), t \in J,
\end{aligned}
$$

where

$$
\begin{aligned}
p(x(\cdot))= & x_{b}-T(b)[\phi(0)+g(0, \phi)]+g\left(b, \bar{x}_{b}\right) \\
& +\int_{0}^{b} A T(b-s) g\left(s, \bar{x}_{s}\right) d s \\
- & \int_{0}^{b} T(b-s) f\left(s, \bar{x}_{\rho\left(s, \bar{x}_{s}\right)}\right) d s-\sum_{k=1}^{m} T\left(b-t_{k}\right) I_{k}\left(\bar{x}_{t_{k}}\right) .
\end{aligned}
$$

and $\bar{x}:(-\infty, b] \rightarrow X$ is such that $\bar{x}_{0}=\phi$ and $\bar{x}=x$ on $[0, b]$ It will be shown that for all $\alpha>0$ the operator $\psi: Z \rightarrow Z$ has a fixed point.

Step 1. For an arbitrary $\alpha>0$, there exists an $r>0$ such that $\psi(Q) \subset Q$. If this is not true, then there exists an $\alpha>0$ such that for every $r>0$, there exist $x^{r} \in Q$ and $t^{r} \in J$ such that $r<\left\|\psi x^{r}\left(t^{r}\right)\right\|$. For such $\alpha>0$, we find that

$$
\begin{aligned}
r< & \left\|\psi x^{r}\left(t^{r}\right)\right\| \\
\leq & \left\|T\left(t^{r}\right)\right\|[\|\phi(0)\|+\|g(0, \phi)\|]+\left\|g\left(t^{r}, \overline{x_{t}^{r}}\right)\right\| \\
& +\int_{0}^{t^{r}}\left\|A T\left(t^{r}-s\right) g\left(s, \overline{x_{s}^{r}}\right)\right\| d s \\
& +\int_{0}^{t^{r}}\left\|T\left(t^{r}-s\right)\right\|\|B\|\|v(s)\| d s \\
& +\int_{0}^{t^{r}}\left\|T\left(t^{r}-s\right)\right\| \| \mid f\left(s, \overline{x^{r}} \rho\left(s, \overline{x_{s}^{r}}\right) \| d s\right. \\
& +\sum_{0<t_{k}<t}\left\|T\left(t^{r}-t_{k}\right)\right\|\left\|I_{k}\left(\bar{x}_{t_{k}}\right)\right\| \\
\leq & M \bar{H}\|\phi\|_{\mathcal{B}}+M\left\|(-A)^{-\beta}\right\|\left(L_{1}\|\phi\|_{\mathcal{B}}+L_{2}\right) \\
& \left.+\left\|(-A)^{-\beta}\right\|\left(L_{1} \| \overline{x_{t}^{r}}\right)\|\|_{\mathcal{B}}+L_{2}\right) \\
& +\int_{0}^{t^{r}}\left\|(-A)^{1-\beta} T\left(t^{r}-s\right)\right\|\left\|(-A)^{\beta} g\left(s, \overline{x_{s}^{r}}\right)\right\| d s \\
& +\int_{0}^{t^{r}}\left\|T\left(t^{r}-\eta\right)\right\|\|B\|\left\|B^{*}\right\|\left\|T^{*}(b-\eta)\right\|\left\|R\left(\alpha, \Gamma_{0}^{b}\right)\right\| \\
& (\times)\left[\left\|x_{b}\right\|+\|T(b)\|[\|\phi(0)\|+\|g(0, \phi)\|]+\left\|g\left(b, \overline{x_{b}^{r}}\right)\right\|\right.
\end{aligned}
$$




$$
\begin{aligned}
& +\int_{0}^{b}\left\|(-A)^{1-\beta} T(b-s)\right\|\left\|\mid(-A)^{\beta} g\left(s, \overline{x_{s}^{r}}\right)\right\| d s \\
& +\int_{0}^{b}\|T(b-s)\|\left\|f\left(s, \bar{x}_{\rho\left(s, \overline{x_{s}^{r}}\right)}\right)\right\| d s \\
& \left.+\sum_{k=1}^{m}\left\|T\left(b-t_{k}\right)\right\|\left\|\mid I_{k}\left(\bar{x}_{t_{k}}\right)\right\|\right](\eta) d \eta \\
& +\int_{0}^{t^{r}}\left\|T\left(t^{r}-s\right)\right\|\left\|f\left(s, \bar{x}_{\rho\left(s, \overline{x_{s}^{r}}\right)}\right)\right\| d s \\
& +\sum_{0<t_{k}<t}\left\|T\left(t^{r}-t_{k}\right)\right\|\left\|I_{k}\left(\bar{x}_{t_{k}}\right)\right\| \\
& \leq M \bar{H}\|\phi\|_{\mathcal{B}}+M M_{0}\left(L_{1}\|\phi\|_{\mathcal{B}}+L_{2}\right)+M_{0}\left(L_{1}\left\|\overline{x_{t}^{r}}\right\|_{\mathcal{B}}+L_{2}\right) \\
& +\frac{C_{1-\beta} b^{\beta}}{\beta}\left(L_{1}\left\|\overline{x_{s}^{r}}\right\|_{\mathcal{B}}+L_{2}\right)+M^{2} M_{1}^{2} \frac{b}{\alpha}\left[\left\|x_{b}\right\|+M \bar{H}\|\phi\|_{\mathcal{B}}\right. \\
& +M M_{0}\left(L_{1}\|\phi\|_{\mathcal{B}}+L_{2}\right)+M_{0}\left(L_{1}\left\|\overline{x_{b}^{r}}\right\|_{\mathcal{B}}+L_{2}\right) \\
& +\frac{C_{1-\beta} b^{\beta}}{\beta}\left(L_{1}\left\|\overline{x_{s}^{r}}\right\|_{\mathcal{B}}+L_{2}\right)+M \int_{0}^{b}\left\|\overline{x^{r}}{ }_{\rho\left(s,\left(\overline{x^{r}}\right)_{s}\right)}\right\|_{\mathcal{B}} d s \\
& \left.+M \sum_{k=1}^{m} d_{k}\right]+M \int_{0}^{t^{r}}\left\|\overline{x^{r}} \rho\left(s,\left(\overline{x^{r}}\right)_{s}\right)\right\|_{\mathcal{B}} d s+M \sum_{k=1}^{m} d_{k} \\
& \leq M \bar{H}\|\phi\|_{\mathcal{B}}+M M_{0}\left(L_{1}\|\phi\|_{\mathcal{B}}+L_{2}\right) \\
& +M_{0}\left(L_{1}\left(\left(M_{a}+J_{0}^{\phi}\right)\|\phi\|_{\mathcal{B}}+K_{a} r\right)+L_{2}\right) \\
& +\frac{C_{1-\beta} b^{\beta}}{\beta}\left(L_{1}\left(\left(M_{a}+J_{0}^{\phi}\right)\|\phi\|_{\mathcal{B}}+K_{a} r\right)+L_{2}\right) \\
& +M^{2} M_{1}^{2} \frac{b}{\alpha}\left[\left\|x_{b}\right\|+M \bar{H}\|\phi\|_{\mathcal{B}}+M M_{0}\left(L_{1}\|\phi\|_{\mathcal{B}}+L_{2}\right)\right. \\
& +M_{0}\left(L_{1}\left(\left(M_{a}+J_{0}^{\phi}\right)\|\phi\|_{\mathcal{B}}+K_{a} r\right)+L_{2}\right) \\
& +\frac{C_{1-\beta} b^{\beta}}{\beta}\left(L_{1}\left(\left(M_{a}+J_{0}^{\phi}\right)\|\phi\|_{\mathcal{B}}+K_{a} r\right)+L_{2}\right) \\
& \left.+M \int_{0}^{b}\left\|\overline{x^{r}} \rho\left(s,\left(\overline{x^{r}}\right)_{s}\right)\right\|_{\mathcal{B}} d s+M \sum_{k=1}^{m} d_{k}\right] \\
& +M \int_{0}^{t^{r}}\left\|\overline{x^{r}} \rho\left(s,\left(\overline{x^{r}}\right)_{s}\right)\right\|_{\mathcal{B}} d s+M \sum_{k=1}^{m} d_{k}
\end{aligned}
$$

For any $x \in Q$, it follows from Lemma 2 that

$$
\left\|{\overline{x^{r}}}_{\rho\left(s,\left(\overline{x^{r}}\right)_{s}\right)}\right\| \leq\left(M_{a}+J_{0}^{\phi}\right)\|\phi\|_{\mathcal{B}}+K_{a} r=r^{*},
$$

where $r^{*}$ is a positive constant. Hence we obtain

$$
\begin{aligned}
\leq & M \bar{H}\|\phi\|_{\mathcal{B}}+M M_{0}\left(L_{1}\|\phi\|_{\mathcal{B}}+L_{2}\right)+M_{0}\left(L_{1} r^{*}+L_{2}\right) \\
& +\frac{C_{1-\beta} b^{\beta}}{\beta}\left(L_{1} r^{*}+L_{2}\right)+M^{2} M_{1}^{2} \frac{b}{\alpha}\left[\left\|x_{b}\right\|+M \bar{H}\|\phi\|_{\mathcal{B}}\right. \\
& +M M_{0}\left(L_{1}\|\phi\|_{\mathcal{B}}+L_{2}\right)+M_{0}\left(L_{1} r^{*}+L_{2}\right) \\
& \left.+\frac{C_{1-\beta} b^{\beta}}{\beta}\left(L_{1} r^{*}+L_{2}\right)+M \int_{0}^{b} \lambda_{r^{*}}(s) d s+M \sum_{k=1}^{m} d_{k}\right] \\
& +M \int_{0}^{t^{r}} \lambda_{r^{*}}(s) d s+M \sum_{k=1}^{m} d_{k}
\end{aligned}
$$

$$
\begin{aligned}
\leq & M \bar{H}\|\phi\|_{\mathcal{B}}+M M_{0}\left(L_{1}\|\phi\|_{\mathcal{B}}+L_{2}\right)+M_{0}\left(L_{1} r^{*}+L_{2}\right) \\
& +\frac{C_{1-\beta} b^{\beta}}{\beta}\left(L_{1} r^{*}+L_{2}\right)+M^{2} M_{1}^{2} \frac{b}{\alpha}\left[K+M_{0}\left(L_{1} r^{*}+L_{2}\right)\right. \\
& \left.+\frac{C_{1-\beta} b^{\beta}}{\beta}\left(L_{1} r^{*}+L_{2}\right)+M \int_{0}^{b} \lambda_{r^{*}}(s) d s+M \sum_{k=1}^{m} d_{k}\right] \\
& +M \int_{0}^{t^{r}} \lambda_{r^{*}}(s) d s+M \sum_{k=1}^{m} d_{k}
\end{aligned}
$$

where $K=\left\|x_{b}\right\|+M \bar{H}\|\phi\|_{\mathcal{B}}+M M_{0}\left(L_{1}\|\phi\|_{\mathcal{B}}+L_{2}\right)+$ $M \sum_{k=1}^{m} d_{k}$

$$
\begin{aligned}
r \leq & \left(M_{0}+\frac{C_{1-\beta} b^{\beta}}{\beta}\right)\left(L_{1} r^{*}+L_{2}\right) \\
& +M^{2} M_{1}^{2} \frac{b}{\alpha}\left(M_{0}+\frac{C_{1-\beta} b^{\beta}}{\beta}\right)\left(L_{1} r^{*}+L_{2}\right) \\
& +M \int_{0}^{b} \lambda_{r^{*}}(s) d s+M^{2} M_{1}^{2} \frac{b}{\alpha} M \int_{0}^{b} \lambda_{r^{*}}(s) d s+K^{*},
\end{aligned}
$$

where $K^{*}=M \bar{H}\|\phi\|_{\mathcal{B}}+M M_{0}\left(L_{1}\|\phi\|_{\mathcal{B}}+L_{2}\right)$

$$
\begin{aligned}
& +M^{2} M_{1}^{2} \frac{b}{\alpha} K+M \sum_{k=1}^{m} d_{k} \\
r \leq & \left(1+M^{2} M_{1}^{2} \frac{b}{\alpha}\right)\left(M_{0}+\frac{C_{1-\beta} b^{\beta}}{\beta}\right)\left(L_{1} r^{*}+L_{2}\right) \\
& +\left(1+M^{2} M_{1}^{2} \frac{b}{\alpha}\right) M \int_{0}^{b} \lambda_{r^{*}}(s) d s+K^{*} \\
\leq & \left(1+M^{2} M_{1}^{2} \frac{b}{\alpha}\right)\left[\left(M_{0}+\frac{C_{1-\beta} b^{\beta}}{\beta}\right)\left(L_{1} r^{*}+L_{2}\right)\right. \\
& \left.+M \int_{0}^{b} \lambda_{r^{*}}(s) d s\right]+K^{*} .
\end{aligned}
$$

We note that $K^{*}$ is independent of $r$ and $r^{*} \rightarrow \infty$ as $r \rightarrow \infty$. Now $\lim \inf _{r \rightarrow \infty} \int_{0}^{b} \frac{\lambda_{r^{*}}(s)}{r} d s=\lim \inf _{r \rightarrow \infty} \int_{0}^{b}\left(\frac{\lambda_{r^{*}}(s)}{r^{*}} \cdot \frac{r^{*}}{r}\right) d s=\Lambda K_{a}$.

Hence we have for $\alpha>0$,

$$
1 \leq\left(1+M^{2} M_{1}^{2} \frac{b}{\alpha}\right)\left[\left(M_{0}+\frac{C_{1-\beta} b^{\beta}}{\beta}\right) L_{1}+\Lambda M\right] K_{a},
$$

which is contradiction to our assumption. Thus $\alpha>0$, there exists an $r>0$ such that $\psi$ maps $Q$ into itself.

Step 2. For each $\alpha>0$, the operator $\psi$ maps $Q$ into a relatively compact subset of $Q$. First, we prove that the set $V(t)=$ $\{\psi x(t) ; x \in Q\}$ is relatively compact in $X$ for every $t \in J$. The case $t=0$ is obvious. So, let $t$ be a fixed real number, and let $\tau$ be a given real number satisfying $0<\tau<t \leq b$, we define

$$
\begin{aligned}
\left(\psi^{\tau} x\right)(t)= & T(t)[\phi(0)+g(0, \phi)]-g\left(t, \bar{x}_{t}\right) \\
& -\int_{0}^{t-\tau} A T(t-s) g\left(s, \bar{x}_{s}\right) d s \\
& +\int_{0}^{t-\tau} T(t-s) B v(s) d s \\
+ & \int_{0}^{t-\tau} T(t-s) f\left(s, \bar{x}_{\rho\left(s, \bar{x}_{s}\right)}\right) d s \\
& +\sum_{0<t_{k}<t} T\left(t-t_{k}\right) I_{k}\left(\bar{x}_{t_{k}}\right) .
\end{aligned}
$$


The set $V_{\tau}(t)=\left\{\left(\psi^{\tau} x\right)(t): x(\cdot) \in Q\right\}$ is relatively compact set in $X$. That is, a finite set $\left\{y_{i}, 1 \leq i \leq n\right\}$ in $X$ exists such that

$$
V_{\tau}(t) \subset \bigcup_{i=1}^{n} \bar{W}\left(y_{i}, \epsilon / 2\right),
$$

where $\bar{W}\left(y_{i}, \epsilon / 2\right)$ is an open ball in $X$ with center at $y_{i}$ and radius $\epsilon / 2$.

On the other hand,

$$
\begin{aligned}
& \left\|(\psi x)(t)-\left(\psi^{\tau} x\right)(t)\right\| \\
& \leq \| \int_{t-\tau}^{t} A T(t-s) g\left(s, \bar{x}_{s}\right) d s+\int_{t-\tau}^{t} T(t-s)[B v(s) \\
& \left.\quad+f\left(s, \bar{x}_{\rho\left(s, \bar{x}_{s}\right)}\right)\right] d s \| \\
& \leq \int_{t-\tau}^{t}\left\|A T(t-s) g\left(s, \bar{x}_{s}\right)\right\| d s+\int_{t-\tau}^{t}\|T(t-\eta)\|
\end{aligned}
$$$$
(\times)\|B\|\left\|B^{*}\right\|\left\|T^{*}(b-\eta)\right\|\left\|R\left(\alpha, \Gamma_{0}^{b}\right)\right\|\left[\left\|x_{b}\right\|+\|T(b)\|[\| \phi(0)\right.
$$$$
+g(0, \phi) \|]+\left\|g\left(b, \bar{x}_{b}\right)\right\|+\int_{0}^{b}\left\|A T(b-s) g\left(s, \bar{x}_{s}\right)\right\| d s
$$$$
+\int_{0}^{b}\|T(b-s)\|\left\|\mid f\left(s, \bar{x}_{\rho\left(s, \bar{x}_{s}\right)}\right)\right\| d s
$$$$
\left.+\sum_{k=1}^{m}\left\|T\left(b-t_{k}\right)\right\|\left\|I_{k}\left(\bar{x}_{t_{k}}\right)\right\|\right] \eta d \eta
$$$$
+\int_{t-\tau}^{t}\|T(t-s)\|\|\| f\left(s, \bar{x}_{\rho\left(s, \bar{x}_{s}\right)}\right) \| d s
$$$$
\leq \int_{t-\tau}^{t} \frac{C_{1-\beta}}{(t-s)^{1-\beta}}\left(L_{1}\left\|\bar{x}_{s}\right\|_{\mathcal{B}}+L_{2}\right) d s
$$$$
+M^{2} M_{1}^{2} \frac{1}{\alpha} \int_{t-\tau}^{t}\left[\left\|x_{b}\right\|+M \bar{H}\|\phi\|_{\mathcal{B}}\right.
$$$$
+M M_{0}\left(L_{1}\|\phi\|_{\mathcal{B}}+L_{2}\right)
$$$$
+M_{0}\left(L_{1}\left\|\bar{x}_{b}\right\|_{\mathcal{B}}+L_{2}\right)+\frac{C_{1-\beta} b^{\beta}}{\beta}\left(L_{1}\left\|\bar{x}_{s}\right\|_{\mathcal{B}}+L_{2}\right)
$$$$
\left.+M \int_{0}^{b}\left\|\bar{x}_{\left(s, \bar{x}_{s}\right)}\right\|_{\mathcal{B}} d s+M \sum_{k=1}^{m} d_{k}\right] d s
$$$$
+M \int_{t-\tau}^{t}\left\|{\overline{x^{r}}}_{\rho\left(s, \bar{x}_{s}\right)}\right\|_{\mathcal{B}} d s
$$$$
\leq \frac{C_{1-\beta} \tau^{\beta}}{\beta}\left(L_{1} r^{*}+L_{2}\right)+M^{2} M_{1}^{2} \frac{1}{\alpha} \int_{t-\tau}^{t}\left[\left\|x_{b}\right\|\right.
$$$$
+M \bar{H}\|\phi\|_{\mathcal{B}}
$$$$
+M M_{0}\left(L_{1}\|\phi\|_{\mathcal{B}}+L_{2}\right)+M_{0}\left(L_{1} r^{*}+L_{2}\right)
$$$$
+\frac{C_{1-\beta} b^{\beta}}{\beta}\left(L_{1} r^{*}+L_{2}\right)
$$$$
\left.+M \int_{0}^{b} \lambda_{r^{*}}(s) d s+M \sum_{k=1}^{m} d_{k}\right] d s+M \int_{t-\tau}^{t} \lambda_{r^{*}}(s) d s
$$$$
\leq \frac{C_{1-\beta} \tau^{\beta}}{\beta}\left(L_{1} r^{*}+L_{2}\right)+M^{2} M_{1}^{2} \frac{1}{\alpha} \int_{t-\tau}^{t}[K
$$$$
+M_{0}\left(L_{1} r^{*}+L_{2}\right)
$$$$
\left.+\frac{C_{1-\beta} b^{\beta}}{\beta}\left(L_{1} r^{*}+L_{2}\right)+M \int_{0}^{b} \lambda_{r^{*}}(s) d s\right] d s
$$

$$
\begin{aligned}
& +M \int_{t-\tau}^{t} \lambda_{r^{*}}(s) d s \\
\leq & \frac{C_{1-\beta} \tau^{\beta}}{\beta}\left(L_{1} r^{*}+L_{2}\right)+M^{2} M_{1}^{2} \frac{1}{\alpha}\left[K+M_{0}\left(L_{1} r^{*}\right.\right. \\
& \left.+L_{2}\right)+\frac{C_{1-\beta} b^{\beta}}{\beta}\left(L_{1} r^{*}+L_{2}\right) \\
& \left.+M \int_{0}^{b} \lambda_{r^{*}}(s) d s\right] \tau+M \int_{t-\tau}^{t} \lambda_{r^{*}}(s) d s \\
\leq & \frac{\epsilon}{2} .
\end{aligned}
$$

Consequently,

$$
V(t) \subset \bigcup_{i=1}^{n} \bar{W}\left(y_{i}, \epsilon\right) .
$$

Hence for each $t \in[0, b], V(t)$ is relatively compact in $X$.

Step 3. $\psi$ maps $Q$ into equicontinuous family. We now show that the set $V=\{(\psi x)(\cdot) \mid x(\cdot) \in Q\}$ is equicontinuous on $[0, b]$. For $0<t_{1}<t_{2} \leq b$, we have

$$
\begin{aligned}
& || z\left(t_{1}\right)-z\left(t_{2}\right) \| \\
& \leq\left\|T\left(t_{1}\right)-T\left(t_{2}\right)\right\|\|\phi(0)+g(0, \phi)\| \\
& +\left\|g\left(t_{1}, \bar{x}_{t_{1}}\right)-g\left(t_{2}, \bar{x}_{t_{2}}\right)\right\| \\
& +\int_{0}^{t_{1}}\left\|T\left(t_{2}-s\right)-T\left(t_{1}-s\right)\right\|\|\| A g\left(s, \bar{x}_{s}\right) \| d s \\
& +\int_{t_{1}}^{t_{2}}\left\|A T\left(t_{2}-s\right)\right\|\left\|g\left(s, \bar{x}_{s}\right)\right\| d s \\
& +\int_{0}^{t_{1}}\left\|T\left(t_{2}-s\right)-T\left(t_{1}-s\right)\right\|\|\| B\|\| v(s) \| d s \\
& +\int_{t_{1}}^{t_{2}}\left\|T\left(t_{2}-s\right)\right\|\|\mid B\|\|v(s)\| d s \\
& +\int_{0}^{t_{1}}\left\|T\left(t_{2}-s\right)-T\left(t_{1}-s\right)\left|\left\||| f\left(s, \bar{x}_{\rho\left(s, \bar{x}_{s}\right)}\right)\right\| d s\right.\right. \\
& +\int_{t_{1}}^{t_{2}}\left\|A T\left(t_{2}-s\right)\right\|\left\|\mid f\left(s, \bar{x}_{\rho\left(s, \bar{x}_{s}\right)}\right)\right\| d s \\
& +\sum_{0<t_{k}<t_{1}}\left\|T\left(t_{2}-t_{k}\right)-T\left(t_{1}-t_{k}\right)\left|\left\||| I_{k}\left(\bar{x}_{t_{k}}\right)\right\|\right.\right. \\
& +\sum_{t_{1}<t_{k}<t_{2}}\left\|T\left(t_{2}-t_{k}\right)\right\|\left\|\mid I_{k}\left(\bar{x}_{t_{k}}\right)\right\| \\
& \leq\left\|T\left(t_{1}\right)-T\left(t_{2}\right)\right\|\|\phi(0)+g(0, \phi)\| \\
& +L_{g}\left[\left(\left|t_{1}-t_{2}\right|+\left\|\bar{x}_{t_{1}}-\bar{x}_{t_{2}}\right\|_{\mathcal{B}}\right)\right] \\
& +\int_{0}^{t_{1}}\left\|T\left(t_{2}-s\right)-T\left(t_{1}-s\right)\right\| N_{0}\left(L_{1}\left\|\bar{x}_{s}\right\|_{\mathcal{B}}+L_{2}\right) d s \\
& +\int_{t_{1}}^{t_{2}} M N_{0}\left(L_{1}\left\|\bar{x}_{s}\right\|_{\mathcal{B}}+L_{2}\right) d s \\
& +M M_{1}^{2} \frac{1}{\alpha} \int_{0}^{t_{1}}\left\|T\left(t_{2}-\eta\right)-T\left(t_{1}-\eta\right)\right\|\left[\left\|x_{b}\right\|\right. \\
& +M \bar{H}\|\phi\|_{\mathcal{B}}+M M_{0}\left(L_{1}\|\phi\|_{\mathcal{B}}+L_{2}\right)
\end{aligned}
$$




$$
\begin{aligned}
& +M_{0}\left(L_{1}\left\|\bar{x}_{b}\right\|_{\mathcal{B}}+L_{2}\right)+\frac{C_{1-\beta} b^{\beta}}{\beta}\left(L_{1}\left\|\bar{x}_{s}\right\|_{\mathcal{B}}+L_{2}\right) \\
& \left.+M \int_{0}^{b}\left\|\bar{x}_{\rho\left(s, \bar{x}_{s}\right)}\right\|_{\mathcal{B}} d s+M \sum_{k=1}^{m} d_{k}\right] d \eta \\
& +M^{2} M_{1}^{2} \frac{1}{\alpha} \int_{t_{1}}^{t_{2}}\left[\left\|x_{b}\right\|+M \bar{H}\|\phi\|_{\mathcal{B}}\right. \\
& +M M_{0}\left(L_{1}\|\phi\|_{\mathcal{B}}+L_{2}\right)+M_{0}\left(L_{1}\left\|\bar{x}_{s}\right\|_{\mathcal{B}}+L_{2}\right) \\
& +\frac{C_{1-\beta} b^{\beta}}{\beta}\left(L_{1}\left\|\bar{x}_{s}\right\|_{\mathcal{B}}+L_{2}\right)+M \int_{0}^{b}\left\|\bar{x}_{\rho\left(s, \bar{x}_{s}\right)}\right\|_{\mathcal{B}} d s \\
& \left.+M \sum_{k=1}^{m} d_{k}\right] d \eta \\
& +\int_{0}^{t_{1}}\left\|T\left(t_{2}-s\right)-T\left(t_{1}-s\right)\left|\left\||| \bar{x}_{\rho\left(s, \bar{x}_{s}\right)}\right\|_{\mathcal{B}} d s\right.\right. \\
& +M \int_{t_{1}}^{t_{2}}\left\|\bar{x}_{\rho\left(s, \bar{x}_{s}\right)}\right\|_{\mathcal{B}} d s+M \sum_{t_{1}<t_{k}<t_{2}} d_{k} \\
& +\sum_{0<t_{k}<t_{1}}\left\|T\left(t_{2}-t_{k}\right)-T\left(t_{1}-t_{k}\right)\right\| d_{k} \\
& \leq\left\|T\left(t_{1}\right)-T\left(t_{2}\right)\right\|\|\|(0)+g(0, \phi) \| \\
& +L_{g}\left[\left(\left|t_{1}-t_{2}\right|+|| \bar{x}_{t_{1}}-\bar{x}_{t_{2}} \|_{\mathcal{B}}\right)\right] \\
& +\int_{0}^{t_{1}}\left\|T\left(t_{2}-s\right)-T\left(t_{1}-s\right)\right\| N_{0}\left(L_{1} r^{*}+L_{2}\right) d s \\
& +\int_{t_{1}}^{t_{2}} M N_{0}\left(L_{1} r^{*}+L_{2}\right) d s \\
& +M M_{1}^{2} \frac{1}{\alpha} \int_{0}^{t_{1}}\left\|T\left(t_{2}-\eta\right)-T\left(t_{1}-\eta\right)\right\|\left[\left\|x_{b}\right\|\right. \\
& +M \bar{H}\|\phi\|_{\mathcal{B}}+M M_{0}\left(L_{1}\|\phi\|_{\mathcal{B}}+L_{2}\right) \\
& +M_{0}\left(L_{1} r^{*}+L_{2}\right)+\frac{C_{1-\beta} b^{\beta}}{\beta}\left(L_{1} r^{*}+L_{2}\right) \\
& \left.+M \int_{0}^{b} \lambda_{r^{*}}(s) d s+M \sum_{k=1}^{m} d_{k}\right] d \eta \\
& +M^{2} M_{1}^{2} \frac{1}{\alpha} \int_{t_{1}}^{t_{2}}\left[\left\|x_{b}\right\|+M \bar{H}\|\phi\|_{\mathcal{B}}+M M_{0}\left(L_{1}\|\phi\|_{\mathcal{B}}+L_{2}\right)\right. \\
& +M_{0}\left(L_{1} r^{*}+L_{2}\right)+\frac{C_{1-\beta} b^{\beta}}{\beta}\left(L_{1} r^{*}+L_{2}\right) \\
& \left.+M \int_{0}^{b} \lambda_{r^{*}}(s) d s+M \sum_{k=1}^{m} d_{k}\right] d \eta \\
& +\int_{0}^{t_{1}}\left\|T\left(t_{2}-s\right)-T\left(t_{1}-s\right)\right\| \lambda_{r^{*}}(s) d s \\
& +M \int_{t_{1}}^{t_{2}} \lambda_{r^{*}}(s) d s+\sum_{0<t_{k}<t_{1}}\left\|T\left(t_{2}-t_{k}\right)-T\left(t_{1}-t_{k}\right)\right\| d_{k} \\
& +M \sum_{t_{1}<t_{k}<t_{2}} d_{k} .
\end{aligned}
$$

The right hand side does not depend on any particular choices of $x(\cdot) \in Q$ and tends to zero as $t_{1}-t_{2} \rightarrow 0$, since the compactness of $T(t)$ for $t>0$ implies the continuity in the uniform operator topology. This proves that $V$ is right equicontinuous at $t \in(0, b)$. The other cases' right equicontinuity at zero and left equicontinuity at $t \in(0, b]$ are similar. Thus $\psi$ maps $Q$ into an equicontinuous family of functions.

Step 4. The map $\psi$ is continuous on $Q$.

Let $\left\{x^{n}\right\}_{n \in N}$ be a sequence in $Q$ and $x \in Q$ such that $x^{n} \rightarrow x$ in $P C$. From $\left(\mathrm{H}_{2}\right)-\left(\mathrm{H}_{6}\right)$, we have

(i) $I_{k}, k=1,2, \ldots, n$ is continuous.

(ii) $g\left(t, \bar{x}_{t}^{n}\right) \rightarrow g\left(t, \bar{x}_{t}\right)$ for each $t \in J$ and since

$$
\left\|g\left(t, \bar{x}_{t}^{n}\right)-g\left(t, \bar{x}_{t}\right)\right\|<2 M_{0} L_{g} r^{*} .
$$

(iii) $A g\left(s, \bar{x}_{t}^{n}\right) \rightarrow A g\left(s, \bar{x}_{t}\right)$ for each $s \in J$ and since

$$
A g\left(s, \bar{x}_{t}^{n}\right)-A g\left(s, \bar{x}_{t}\right) \|<2 N_{0} L_{g} r^{*} .
$$

(iv) $f\left(s, \bar{x}_{\rho\left(s, \bar{x}_{s}\right)}^{n}\right) \rightarrow f\left(s, \bar{x}_{\rho\left(s, \bar{x}_{s}\right)}\right)$ for each $s \in J$ and since

$$
\left\|f\left(s, \bar{x}_{\rho\left(s, \bar{x}_{s}\right)}^{n}\right)-f\left(s, \bar{x}_{\rho\left(s, \bar{x}_{s}\right)}\right)\right\|<2 \lambda_{r^{*}}(s) .
$$

From the Lebesgue dominated convergence theorem, we obtain

$$
\begin{aligned}
& \left\|\psi z^{n}-\psi z\right\| \\
& \leq\left\|g\left(t, \bar{x}_{t}^{n}\right)-g\left(t, \bar{x}_{t}\right)\right\| \\
& +\int_{0}^{t}\left\|A T(t-s) g\left(s, \bar{x}_{s}^{n}\right)-A T(t-s) g\left(s, \bar{x}_{s}\right)\right\| d s \\
& +\int_{0}^{t}\left\|T ( t - \eta ) \left|\left\|| | B \left|\left\|| | B ^ { * } | | | | T ^ { * } ( b - \eta ) \left|\left\||| R\left(\alpha, \Gamma_{0}^{b}\right)\right\|\right.\right.\right.\right.\right.\right. \\
& (\times)\left[\left\|g\left(b, \bar{x}_{b}^{n}\right)-g\left(b, \bar{x}_{b}\right)\right\|\right. \\
& +\int_{0}^{b}\|T(b-s)\|\left\|\mid A g\left(s, \bar{x}_{s}^{n}\right)-A g\left(s, \bar{x}_{s}\right)\right\| d s \\
& +\int_{0}^{b}\|T(b-s)\|\left\|\mid f\left(s, \bar{x}_{\rho\left(s, \bar{x}_{s}\right)}^{n}\right)-f\left(s, \bar{x}_{\rho\left(s, \bar{x}_{s}\right)}\right)\right\| d s \\
& +\sum_{0<t_{k}<t}\left\|T\left(b-t_{k}\right)\left|\left\||| I_{k}\left(\bar{x}_{t_{k}}^{n}\right)-I_{k}\left(\bar{x}_{t_{k}}\right)\right\|\right](\eta) d \eta\right. \\
& +\int_{0}^{t}\|T(t-s)\|\|\| f\left(s, \bar{x}_{\rho\left(s, \bar{x}_{s}\right)}^{n}\right)-f\left(s, \bar{x}_{\rho\left(s, \bar{x}_{s}\right)}\right) \| d s \\
& +\sum_{0<t_{k}<t}\left\|T\left(t-t_{k}\right)\right\|\left\|I_{k}\left(\bar{x}_{t_{k}}^{n}\right)-I_{k}\left(\bar{x}_{t_{k}}\right)\right\| \\
& \leq\left\|g\left(t, \bar{x}_{t}^{n}\right)-g\left(t, \bar{x}_{t}\right)\right\| \\
& +M \int_{0}^{t}\left\|A g\left(s, \bar{x}_{s}^{n}\right)-A g\left(s, \bar{x}_{s}\right)\right\| d s \\
& +M M_{1}^{2} \frac{1}{\alpha} \int_{0}^{b}\left[\left\|g\left(b, \bar{x}_{b}^{n}\right)-g\left(b, \bar{x}_{b}\right)\right\|\right. \\
& +M \int_{0}^{t}\left\|A g\left(s, \bar{x}_{s}^{n}\right)-A g\left(s, \bar{x}_{s}\right)\right\| d s \\
& +M \int_{0}^{b}\left\|f\left(s, \bar{x}_{\rho\left(s, \bar{x}_{s}\right)}^{n}\right)-f\left(s, \bar{x}_{\rho\left(s, \bar{x}_{s}\right)}\right)\right\| d s \\
& \left.+M \sum_{0<t_{k}<t}\left\|I_{k}\left(\bar{x}_{t_{k}}^{n}\right)-I_{k}\left(\bar{x}_{t_{k}}\right)\right\|\right] d \eta \\
& +M \int_{0}^{t}\left\|f\left(s, \bar{x}_{\rho\left(s, \bar{x}_{s}\right)}^{n}\right)-f\left(s, \bar{x}_{\rho\left(s, \bar{x}_{s}\right)}\right)\right\| d s \\
& +M \sum_{0<t_{k}<t}\left\|I_{k}\left(\bar{x}_{t_{k}}^{n}\right)-I_{k}\left(\bar{x}_{t_{k}}\right)\right\|
\end{aligned}
$$


$\rightarrow 0$ as $n \rightarrow \infty$. Which shows the continuity of $\psi$.

Hence all the conditions of Schauder's fixed point theorem [27] are satisfied, and consequently the operator $\psi$ has a fixed point in $Q$. Thus the problem (1) - (3) has a solution on $J$.

THEOREM 2. Assume that linear system (4) - (5) is approximately controllable on $J$. If the conditions $\left(H_{4}\right),\left(H_{6}\right),\left(H_{7}\right)$ and $\left(S_{1}\right)$ are satisfied then the system (1) - (3) is approximately controllable.

Proof. Let $x_{\alpha}(\cdot)$ be a fixed point of $\psi$ in $Q$ any fixed point of $\psi$ is a mild solution of 1 - 3 under the control

$$
u_{\alpha}(t)=B^{*} T^{*}(b-t) R\left(\alpha, \Gamma_{0}^{b}\right) p\left(x_{\alpha}\right)
$$

and satisfies the inequality

$$
x_{\alpha}(b)=x_{b}+\alpha R\left(\alpha, \Gamma_{0}^{b}\right) p\left(x_{\alpha}\right) .
$$

By the conditions $\left(H_{4}\right)$ and $\left(H_{7}\right)$,

$$
\begin{gathered}
\int_{0}^{b} \| f\left(s, \bar{x}_{\rho\left(s, \bar{x}_{s}^{\alpha}\right)}^{\alpha} \|^{2} d s \leq b N^{2},\right. \\
\int_{0}^{b}\left\|(-A)^{\beta} g\left(s, \bar{x}_{s}^{\alpha}\right)\right\|^{2} d s \leq b \bar{N}^{2} .
\end{gathered}
$$

Consequently, the sequence $\left\{f\left(s, \bar{x}_{\rho\left(s, \bar{x}_{s}^{\alpha}\right)}^{\alpha}\right)\right\} \quad$ and $\left\{(-A)^{\beta} g\left(s, \bar{x}_{s}^{\alpha}\right)\right\}$ is bounded in $L_{2}(J, X)$. Then there is a subsequence, still denoted by $\left\{f\left(s, \bar{x}_{\rho\left(s, \bar{x}_{s}^{\alpha}\right)}^{\alpha}\right)\right\}$ and $\left\{(-A)^{\beta} g\left(s, \bar{x}_{s}^{\alpha}\right)\right\}$ that weakly converges to, say, $f(s)$ and $g(s)$ in $L_{2}(J, X)$ respectively. Define

$$
\begin{aligned}
p(x(\cdot))= & x_{b}-T(b)[\phi(0)+g(0, \phi)]+g\left(b, \bar{x}_{b}\right) \\
& +\int_{0}^{b} A T(b-s) g\left(s, \bar{x}_{s}^{\alpha}\right) d s \\
- & \int_{0}^{b} T(b-s) f\left(s, \bar{x}_{\rho\left(s, \bar{x}_{s}^{\alpha}\right)}^{\alpha}\right) d s-\sum_{k=1}^{m} T\left(b-t_{k}\right) I_{k}\left(\bar{x}_{t_{k}}\right), \\
w= & x_{b}-T(b)[\phi(0)+g(0, \phi)]+g\left(b, \bar{x}_{b}\right) \\
& +\int_{0}^{b} A T(b-s) g(s) d s-\int_{0}^{b} T(b-s) f(s) d s \\
- & \sum_{k=1}^{m} T\left(b-t_{k}\right) I_{k}\left(\bar{x}_{t_{k}}\right) .
\end{aligned}
$$

It follows by the compactness of the operators $l(\cdot) \rightarrow$ $\int_{0}^{\cdot}(-A)^{-\beta} T(\cdot-s) l(s) d s: L_{2}(J, X) \rightarrow C(J, X)$ and $l(\cdot) \rightarrow$ $\int_{0}^{\cdot}(-A) T(\cdot-s) l(s) d s: L_{2}(J, X) \rightarrow C(J, X)$ we obtain that

$$
\begin{aligned}
& \left\|p\left(x_{\alpha}\right)-w\right\| \\
& =\left\|\int_{0}^{b}(-A)^{-\beta} T(b-s)\left[(-A)^{\beta} g\left(s, \bar{x}_{s}^{\alpha}\right)-g(s)\right] d s\right\| \\
& +\left\|\int_{0}^{b} T(b-s)\left[f\left(s, \bar{x}_{\rho\left(s, \bar{x}_{s}^{\alpha}\right)}^{\alpha}\right)-f(s)\right] d s\right\| \rightarrow 0 \\
& \text { as } \quad \alpha \rightarrow 0^{+} .
\end{aligned}
$$

Then from (6), we obtain

$$
\begin{aligned}
& \left\|x_{\alpha}(b)-x_{b}\right\| \\
& =\left\|\alpha R\left(\alpha, \Gamma_{0}^{b}\right) p\left(x_{\alpha}\right)\right\| \\
& =\left\|\alpha R\left(\alpha, \Gamma_{0}^{b}\right)\left(p\left(x_{\alpha}\right)-w+w\right)\right\| \\
& \leq\left\|\alpha R\left(\alpha, \Gamma_{0}^{b}\right)(w)\right\|+\left\|\alpha R\left(\alpha, \Gamma_{0}^{b}\right)\right\|\left\|\left(p\left(x_{\alpha}\right)-w\right)\right\| \\
& \leq\left\|\alpha R\left(\alpha, \Gamma_{0}^{b}\right)(w)\right\|+\left\|\left(p\left(x_{\alpha}\right)-w\right)\right\| \rightarrow 0
\end{aligned}
$$

as $\alpha \rightarrow 0^{+}$. This completes the approximate controllability of 1 (3).

\section{CONCLUSION}

Sufficient conditions for approximate controllability results are established for a class of impulsive neutral functional differential equation with state-dependent delay. The proof of the main theorem is based on the application of the Schauder's fixed point theorem and fractional powers of operators with semigroup theory.

\section{REFERENCES}

[1] Y. V. Rogovchenko, Impulsive evolution systems: Main results and new trends, Dynam. Contin.,Discrete Impuls. Systems, 3 (1997), 57-88.

[2] M. Benchohra, J. Henderson and S. K. Ntouyas, Impulsive Differential Equations and Inclusions, Series Contemporary Mathematics and Its Applications, Vol. 2, Hindawi Publ. Corp.,2006.

[3] V. Lakshmikantham, D. D. Bainov and P. S. Simeonov, Theory of Impulsive Differential Equations, World Scientific Pub. Co., Singapore, 1989.

[4] A. Samoilenko and N. Peresyuk, Differential Equations with Impusive Effectes, World Scientific Pub. Co., Singapore, 1995.

[5] M. Benchohra, A. Ouahab, Controllability results for functional semilinear differential inclusions in Frechet spaces, Nonlinear Anal. TMA, 61(2005), 405-423.

[6] N. Abada, M. Benchohra and H. Hammouche, Existence and controllability results for nondensely de-ned impulsive semilinear functional differential inclusions, J. Differ. Equ., 246 (2009), 3834-3863.

[7] J. Klamka, Constrained controllability of semilinear systems with delays, Nonlinear Dynam., 56 (2009), 169177.

[8] J. Klamka, Constrained controllability of semilinear systems with delayed controls, Bull. Pol. Ac. Tech., 56 (2008), 333-337.

[9] L. Gorniewicz, S. K. Ntouyasand D. O'Regan, Controllability of semilinear differential equations and inclusions via semigroup theory in Banach spaces, Rep. Math. Phys., 56 (2005), 437-470.

[10] L. Gorniewicz, S. K. Ntouyas and D. O'Regan, Existence and controllability results for first and second order functional semilinear differential inclusions with nonlocal conditions, Numer. Funct. Anal. Optim., 28 (2007), 53-82.

[11] L. Gorniewicz, S. K. Ntouyas, D. O'Regan, Controllability results for -rst and second order evolution inclusions with nonlocal conditions, Ann. Polon. Math., 89 (2006), 65-101. 
[12] N. I. Mahmudov, Approximate controllability of evolution systems with nonlocal Conditions, Nonlinear Anal. TMA, 68 (2008), 536-546.

[13] R. Sakthivel, Y. Ren and N. I. Mahmudov, Approximate controllability of second order stochastic differential equations with impulsive e-ects, Modern Phys. Lett. B 24 (2010), 1559-1572.

[14] R. Sakthivel, J. Juan, Nieto, N. I. Mahmudov, Approximate controllability of nonlinear deterministic and stochastic systems with unbounded delay, Taiwan J. Math., 14 (2010), 1777-1797.

[15] R. Sakthivel, Approximate controllability of impulsive stochastic evolution Equations, Funkcialaj Ekvacioj, 52 (2009), 381-393.

[16] J. Klamka, Constrained approximate controllability, IEEE. T. Automat. Contr., 45 (2000), 1745-1749.

[17] R. Sakthivel and E. R. Anandhi, Approximate controllability of impulsive differential equations with statedependent delay, International Journal of Control, 82, 2 (2010), 387-393.

[18] B. Radhakrishnan and K. Balachandran, Controllability of impulsive neutral functional evolution integrodifferential systems with infinite delay, Nonlinear Analysis, HS, 5 (2011), 655-670.

[19] N. I. Mahmudov, Approximate controllability of semilinear deterministic and stochastic evolution equations in abstract spaces, SIAM J, Control Optim., 42 (2003), 1604-1622.

[20] Y. -K. Chang and W. S. Li, Solvability for impulsive neutral integrodifferential equations with state-dependent delay via fractional operators, J. Optim. Theory Appl., 144 (2010), 445-459.

[21] M. Mallika Arjunan and V. Kavitha, Existence results for impulsive neutral functional differential equations with state-dependent delay, Electronic Journal of Qualitative Theory of Differential equations, 26 (2009), 1-13.

[22] A. Pazy, Semigroups of Linear Operators and Applications to Partial Differential Equations, Springer, New York, (1983).

[23] Y. Heno, S. Murakami and T. Naito, Functional differential equations with infinite delay, Lecturer Notes in Mathematics, 1473, Springer-Verlag, Berlin, 1991.

[24] E. Hernandez, R. Sakthivel and S. Tanaka, Existence results for impulsive evolution equations with statedependent delay, Electro. J. Differential Equations, 28 (2008), 1-11.

[25] A. E. Bashirov and N. I. Mahmudov, On concept of controllability for linear deterministic and stochastic systems, SIAM, J. Control Optim., 37 (1999), 1808-1821.

[26] E. Hernandez, A. Prokopczyk and L. A. Ladeira, A note on partial functional differential equations with statedependent delay, Nonlinear Anal. RWA, 7 (2006), 510519.

[27] A. Granas and J. Dugundji, Fixed Point Theory, Springer, New York, 2003. 